\title{
Sharp transitions of gamma coherence in inhibitory networks occur when a biological context and constraints are imposed
}

\author{
Katie A Ferguson ${ }^{1,2^{*}}$, Carey YL Huh ${ }^{4}$, Bénédicte Amilhon ${ }^{4}$, Sylvain Williams ${ }^{4}$, Frances K Skinner ${ }^{1,3}$ \\ From Twenty Second Annual Computational Neuroscience Meeting: CNS*2013 \\ Paris, France. 13-18 July 2013
}

From several experimental and modeling studies, it has become apparent that networks of parvalbumin-positive $(\mathrm{PV}+)$, fast-firing interneurons play an essential role in generating population gamma rhythms [1]. Many features of these perisomatically-inhibiting PV+ cell networks influence the generation of gamma oscillations, a number of which have been explored in network models [2,3]. These include the synaptic delay, the decay time constant of the postsynaptic conductance, and whether the reversal potential is hyperpolarizing or shunting. While it is clear that all these features affect whether and how much coherence occurs at gamma frequencies, it is much less clear which features (i.e. parameters and parameter balances) may be essential in various biological contexts. To address this, we used our previously constructed network model of hippocampal CA1 PV+ cells [4]. Network size, connectivity, and cellular components would all be expected to affect the network dynamics. Thus, experimental data from an intact hippocampus in vitro was used to obtain a clear biological context, and importantly, the amount of input that these cells receive during ongoing theta population activities was estimated. Using these cellular components and input characteristics, we found that coherent gamma oscillations could emerge within experimental constraints for particular parameter balances. Here we investigate the effect of appropriately sized and connected networks.

Each cell in the network received excitatory input $\left(\mathrm{I}_{\mathrm{ap}}\right.$ plied), which was heterogeneous across cells, and synaptic inhibition from presynaptic interneurons, which had a particular maximal conductance value $\left(\mathrm{g}_{\mathrm{PV}}\right)$. We systematically

\footnotetext{
* Correspondence: kt.ferguson@gmail.com

${ }^{1}$ Toronto Western Research Institute, University Health Network, Toronto,

Ontario, M5T 2S8, Canada

Full list of author information is available at the end of the article
}

varied $g_{P V}$ and $I_{\text {applied }}$ within the experimentally determined ranges, and determined the coherence of the network model population firing and the network frequency for each combination of these values. For each of these network simulation sets, we also varied the connectivity probability as well as network size to explore how these factors affect the network's ability to produce coherent network firing. We find that when our connection probability is similar to what has been found experimentally, our networks exhibit a sharp transition from random firing to network coherence with only a small change in $\mathrm{I}_{\text {applied. }}$. However, as connectivity in the network is increased beyond experimentally estimated values, this sharp transition disappears. Instead, a larger window of coherence is achieved, but with a smooth transition from random to coherent firing. Similarly, decreasing the network size increases the window of coherent firing, and while these sharp transitions remain for changing network sizes, the parameter regimes in which they occur changes significantly when the network size becomes much smaller than experimental estimates. Our work indicates that CA1 fastspiking PV+ networks are capable of producing gamma population rhythms, and that perturbation in and out of coherent states can occur abruptly given the sharp transitions obtained in our network simulations. Since our simulations approximate a biological context, it may be that gating in and out of coherence is a design property to allow generation of theta/gamma network oscillations.

\footnotetext{
Acknowledgements

This work was supported by the Canadian Institutes of Health Research and Natural Sciences and Engineering Research Council of Canada.

Computations were performed on the gpc supercomputer at the SciNet HPC Consortium. SciNet is funded by: the Canada Foundation for Innovation under the auspices of Compute Canada; the Government of Ontario; Ontario Research Fund - Research Excellence; and the University of Toronto.
} 


\section{Author details}

'Toronto Western Research Institute, University Health Network, Toronto, Ontario, M5T 2S8, Canada. ${ }^{2}$ Physiology, University of Toronto, Toronto, Ontario, M5S 1A1, Canada. ${ }^{3}$ Medicine (Neurology), Physiology, Institute of Biomaterials and Biomedical Engineering, University of Toronto, Toronto, Ontario, M5S 1A1, Canada. ${ }^{4}$ Psychiatry, Douglas Mental Health University Institute, McGill University, Montreal, Quebec, H4G 1X6, Canada.

Published: 8 July 2013

\section{References}

1. Buzsáki G, Wang XJ: Mechanisms of gamma oscillations. Annu Rev Neurosci 2012, 35:203-225.

2. Bartos M, Vida I, Jonas P: Synaptic mechanisms of synchronized gamma oscillations in inhibitory interneuron networks. Nat Rev Neurosci 2007, 8:45-56.

3. Wang XJ, Buzsáki G: Gamma oscillation by synaptic inhibition in a hippocampal interneuronal network model. J Neurosci 1996, 16:6402-6413.

4. Ferguson KA, Huh CYL, Amilhon B, Murugesu R, Williams S, Skinner FK: Experimentally constrained network model of hippocampal fast-firing parvalbumin-positive interneurons. BMC Neurosci 2012, 13:05.

doi:10.1186/1471-2202-14-S1-P365

Cite this article as: Ferguson et al:: Sharp transitions of gamma coherence in inhibitory networks occur when a biological context and constraints are imposed. BMC Neuroscience 2013 14(Suppl 1):P365.

\section{Submit your next manuscript to BioMed Central} and take full advantage of:

- Convenient online submission

- Thorough peer review

- No space constraints or color figure charges

- Immediate publication on acceptance

- Inclusion in PubMed, CAS, Scopus and Google Scholar

- Research which is freely available for redistribution

Submit your manuscript at www.biomedcentral.com/submit 